\title{
Fetal Lung Maturity Analysis Using Ultrasound Image Features
}

\author{
K. N. Bhanu Prakash, A. G. Ramakrishnan, Senior Member, IEEE, S. Suresh, and Teresa W. P. Chow
}

\begin{abstract}
This pilot study was carried out to find the feasibility of analyzing the maturity of the fetal lung using ultrasound images. Data were collected from normal pregnant women at intervals of two weeks from the gestation age of 24 to 38 weeks. Images were acquired at two centers located at different geographical locations. The total data acquired consisted of 750 images of immature and 250 images of mature class. A region of interest of $64 \times 64$ pixels was used for extracting the features. Various textural features were computed from the fetal lung and liver images. The ratios of fetal lung to liver feature values were investigated as possible indexes for classifying the images into those from mature (reduced pulmonary risk) and immature (possible pulmonary risk) lung. The features used are fractal dimension, lacunarity, and features derived from the histogram of the images. The following classifiers were used to classify the fetal lung images as belonging to mature or immature lung: nearest neighbor, $k$-nearest neighbor, modified $k$-nearest neighbor, multilayer perceptron, radial basis function network, and support vector machines. The classification accuracy obtained for the testing set ranges from $73 \%$ to $96 \%$.
\end{abstract}

Index Terms-Classification, fetal lung, fractal dimension, lung maturity, sonogram, texture.

\section{INTRODUCTION}

$\mathbf{T}$ HE ASSESSMENT of fetal lung maturity is of great value in perinatal management. The lung development involves biochemical and anatomical components. Biochemical maturity can occur as early as 28 weeks or as late as term. However, the anatomical development is closely related to the gestation age (GA). Congenital anomalies account for $20-25 \%$ of perinatal deaths [1]. Prenatal diagnosis (PD) is essential to avoid an untoward outcome for the fetus or the mother, or both. Specifically, PD helps in managing the remaining weeks of the pregnancy, determining the outcome of the pregnancy, planning for possible complications during birth, planning for problems that may occur in the newborn infant, deciding whether to continue the pregnancy, and finding conditions that may affect future pregnancies. A variety of noninvasive and invasive techniques are available for PD. Each of them can be applied only at specific time periods during the pregnancy for greatest utility. All the techniques for PD except ultrasonography are invasive, involving at least a sample of maternal blood.

In amniocentesis, the study of lecithin-sphingomyelin (L/S) ratio is the most useful. This test, being invasive, carries risks,

Manuscript received October 16, 2000; revised July 26, 2001.

K. N. Bhanu Prakash and A. G. Ramakrishnan are with the Biomedical Laboratory, Department of Electrical Engineering, Indian Institute of Science, Bangalore, 560012 Karnataka, India (e-mail: ramkiag@ee.iisc.ernet.in).

S. Suresh is with the Mediscan Systems, Chennai, India.

T. W. P. Chow is with the Department of Obstetrics and Gynecology, University of Malaya, Kuala Lumpur, Malaysia.

Publisher Item Identifier S 1089-7771(02)02010-1. and on occasion may be contraindicated. Ultrasonography is a noninvasive procedure that is harmless to both the fetus and the mother. Ultrasound can be used to determine fetal size, GA, and the condition of placenta. Ultrasound cannot measure any of the biochemical parameters of fetal lung maturity (LM), nor can it provide direct histologic information about fetal lung development. However, it is reasonable to assume that both morphological and biochemical changes alter the diffuse scattering and other propagation properties of fetal lung. This may change the textural appearance of sonogram. Sonographically determined parameters of fetal biparietal diameter (BPD) and placental grading (PG) have been related to fetal LM, with an accuracy ranging from $78 \%$ to $100 \%$ [2].

There has been an extensive debate for and against the use of sonographic features for analyzing fetal lung maturity. Thieme et al. [3] studied the lung development in a lamb and analyzed the development of lung through sonographic patterns. Garrett et al. [4] state that reflectivity of human fetal lung is equal to or less than that of liver throughout most of pregnancy, but that relationship reverses in late gestation. Cayea et $a l$. [5] claim that there is no statistically significant correlation between the sonographic features and the biochemical fetal LM indexes, namely, L/S ratio and phosphatidylglycerol values. Benson et al. [6] employed radio-frequency (RF) signals for characterizing fetal lung and liver tissues. They observed a spectral shift in the reflected signals from a high to a low frequency range, when the lung makes a transition from immature to mature state. Feingold et al. [2] used densitometer measurements to establish a correlation between lung-liver echogenicity and L/S ratio. Podobnik et al. [7] developed a relation between the coefficient of variation of lung-liver echogenicity and L/S ratio. Petrucha et al. [8] measured BPD and PG from ultrasound. They concluded that ultrasonically determined BPD together with the PG is at least as sensitive as the L/S ratio in predicting the pulmonary maturity. Sohn et al. [9]-[11] carried out frequency analysis of both fetal lung and liver, and the ratio between the mean and the range of the frequencies received was used as an index of maturity analysis.

Some of the earlier studies have used echogenicity of the lung and liver as a possible index of pulmonary maturity [2], [4], [9]. Many researchers have used textural measures to study and analyze the liver texture in differential diagnosis of liver [12], [13]. Since echogenicity is sensitive to machine settings, and textural features have been used in the literature to analyze the echotexture of liver, we have tried in this work to classify lung images as belonging to mature (least or no pulmonary risk) or immature (possible pulmonary risk) classes using the textural features described in Section III. The motivation for the work described in this paper was to find the possible changes in the sonogram tex- 
ture during the transition from a high pulmonary risk group to low risk group. Here we have attempted quantitative texture assessment rather than qualitative (which could be subjective). In this paper, we refer to the group with pulmonary risk as immature class and the one with reduced risk as mature class. Since it is known clinically that many babies at 34 weeks of gestation do not have pulmonary risk, we have considered subjects above 35 weeks as belonging to the mature class.

\section{Data Collection}

Ultrasound examinations were performed both at Mediscan Systems, Chennai, India, and at the University Hospital in Kuala Lumpur, Malaysia. Data were collected in both places using the real-time ATL Apogee 800 plus scanner with a $3.5-\mathrm{MHz}$ curvilinear, broad bandwidth transducer probe with the dynamic range set at $55 \mathrm{~dB}$. Adequate care was taken to ensure that imaging was performed in a similar way at both locations. The overall gain was set at an optimal value to get uniform visibility. Longitudinal and transverse sections of the fetal thorax and upper abdomen were imaged. The fetal lung and liver were identified in the thoracic and upper abdominal sections, respectively. Care was taken to avoid obvious vascular structures in the liver. The machine settings were optimized to obtain a uniform echo texture. The postprocessing curves were unchanged. The focal zone was adjusted so that the area of interest was always in the focal zone. To test the validity of the method across populations, and to confirm its robustness, samples were collected from 1000 subjects belonging to Indian, Chinese, and Malayan races. Data were collected from the subjects at GAs from 24 to 38 weeks, at regular intervals of two weeks. Since most deliveries after 34 weeks can be effectively handled with medical support, and in most Asian races the pregnancy ends around 38 weeks, data were collected only up to 38 weeks. The subjects were rigorously followed up throughout the pregnancy and also after delivery. Only the data corresponding to normal pregnancies, also leading to babies with normal pulmonary functions, were included in our analysis.

The purpose of our pilot study was to obtain the trend of the textural features of the normal fetal lung as a function of the GA. A cross-sectional study of normal fetuses has shown that the overall size of a fetus at each GA falls within a range of fifth to ninety-fifth percentile. Hence, in our study, since normal subjects are chosen, large variations in depth were not encountered. The lung and the liver areas taken for analysis were contiguous and at the same depth for each fetus. The appropriate section of each image was frozen and then transferred to videotape. The images were then digitized using an 8-bit Creative video grabber card. The size of the digitized image was $320 \times 240$ pixels with a resolution of 29 pixels $/ \mathrm{cm}$. The histogram of the images was first stretched to have a uniform range of gray values. To obtain good estimates of the fractal features, it is advisable to get as large a region of interest (ROI) as possible. However, we had the constraint that the same area of ROI must be available in both the liver and the lung regions of all the images obtained. Thus, regions of interest (ROIs) of $64 \times 64$ pixels each from the lung and liver regions were used for extracting textural features. Fig. 1 shows two fetal echogram samples, with the ROI selected from both the liver and the lung regions. The total data

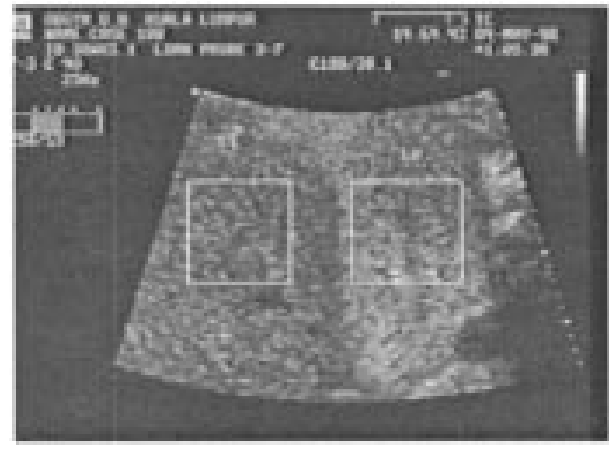

(a)

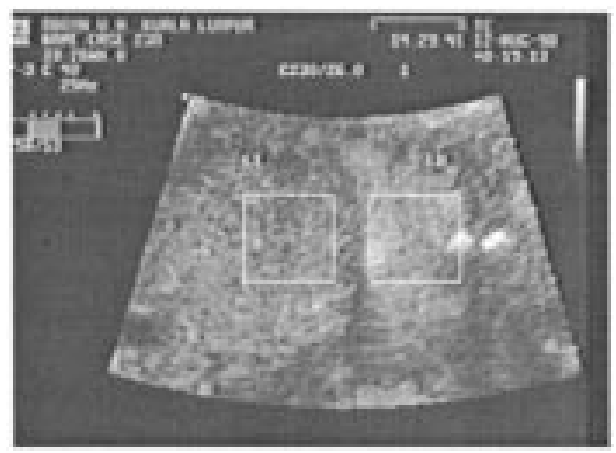

(b)

Fig. 1. Samples of fetal echogram with lung and liver regions and ROIs. (a) 30 weeks and (b) 26 weeks.

acquired consisted of 750 images from immature class and 250 images from mature class. The lung-to-liver ratio of various feature values was studied as a possible index of maturity.

\section{FEATURE EXTRACTION}

The various textural features extracted are described in this section. The features were analyzed using statistical tests to find the correlation with respect to GA. Their computational costs were also considered in finalizing the features to be used for classification. A feature vector was formed using the selected features.

\section{A. Spatial Gray-Level Dependence Matrices (SGLDMs)}

The SGLDMs are based on the estimation of second-order joint conditional probability density functions, denoted by $f(i, j \mid d, \theta)$. Here $f(i, j \mid d, \theta)$ is the probability that a pair of pixels separated by a distance $d$ at an angle $\theta$ have gray levels $i$ and $j$. The angles are quantized to $45^{\circ}$ intervals. The estimated probability density functions, denoted by $P(i, j \mid d, \theta)$, are obtained as in [14] and [15].

Haralick [15] proposed 14 texture measures that can be extracted from the $P(i, j \mid d, \theta)$ matrices. In our study, the five texture features (energy $E$, entropy $H$, correlation $C$, inertia $I_{n}$ and local homogeneity $L$ ) are computed

$$
\begin{aligned}
& E=\sum_{i} \sum_{j}\left[s_{\theta}(i, j \mid d)\right]^{2} \\
& H=-\sum_{i} \sum_{j} \theta(i, j \mid d) \log s_{\theta}(i, j \mid d)
\end{aligned}
$$




$$
\begin{aligned}
C & =\frac{1}{\sigma_{x} \sigma_{y}} \sum_{i} \sum_{j}\left(i-\mu_{x}\right)\left(j-\mu_{y}\right) s_{\theta}(i, j \mid d) \\
I_{n} & =\sum_{i} \sum_{j}(i-j)^{2} s_{\theta}(i, j \mid d) \\
L & =\sum_{i} \sum_{j} \frac{1}{1+(i-j)^{2}} s_{\theta}(i, j \mid d)
\end{aligned}
$$

where $\mu_{x}=\sum_{i} \sum_{j} s_{\theta}(i, j \mid d)$ and $\sigma_{x}^{2}=\sum_{i}\left(i-\mu_{x}\right)^{2}$ $\sum_{j}\left[s_{\theta}(i, j \mid d)\right]$.

The two summations are carried out over all the values of $i \in\left(0, N_{G}-1\right)$ and $j \in\left(0, N_{G}-1\right)$, respectively. $s_{\theta}(i, j \mid d)$ is the $(i, j)$ th element of $S_{\theta}$ for a specified $d, N_{G}$ is the number of gray levels in the image, and $S_{\theta^{\circ}}(d)=P\left(i, j \mid d, \theta^{\circ}\right)$. Similarly, $\mu_{y}$ and $\sigma_{y}$ are computed. Each measure is evaluated for $d=1$ and $\theta=0^{\circ}, 45^{\circ}, 90^{\circ}$, and $135^{\circ}$.

\section{B. Gray-Level Difference Matrix (GLDM)}

Let $I(x, y)$ be the image intensity function. For any given displacement $\delta=(\Delta x, \Delta y)$, let $I_{\delta}(x, y)=\mid I(x, y)-$ $I(x+\Delta x, y+\Delta y) \mid$ and $f^{\prime}(i \mid \delta)$ be the probability density of $I_{\delta}(x, y)$. If there are $m$ gray levels, this has the form of an $m$-dimensional vector whose $i$ th component is the probability that $I_{\delta}(x, y)$ will have value $i$. The value of $f^{\prime}(i \mid \delta)$ is obtained from the number of times $I_{\delta}(x, y)$ occurs for a given $\delta$, i.e., $f^{\prime}(i \mid \delta)=P\left(I_{\delta}(x, y)=i\right)$. Four possible forms of the vector $\delta$ were considered: $(0, d),(-d, d),(d, 0)$, and $(-d,-d)$, where $d$ is the interpixel distance. From each of the density functions, five texture features [contrast (CON), mean, entropy (ENT), inverse difference moment (IDM), and angular second moment (ASM)] were extracted. In each case, the subscript $i \in\left(0, N_{G}-1\right)$

$$
\begin{aligned}
\mathrm{CON} & =\sum_{i} i^{2} f^{\prime}(i \mid \delta) \\
\text { Mean }: & \sum_{i} i f^{\prime}(i \mid \delta) \\
\mathrm{ENT}= & \sum_{i} f^{\prime}(i \mid \delta) \log \left(f^{\prime}(i \mid \delta)\right) \\
\mathrm{IDM}= & \sum_{i} f^{\prime}(i \mid \delta) /\left(i^{2}+1\right) \\
\mathrm{ASM} & =\sum_{i}\left[f^{\prime}(i \mid \delta)\right]^{2} .
\end{aligned}
$$

\section{Laws' Textural Measures}

Laws' textural energy measures [17] are derived from three vectors, each of length three: $L 3=\{1,2,1\}, E 3=$ $\{-1,0,1\}$, and $S 3=\{-1,2,-1\}$. These represent, respectively, the operations of local averaging, edge detection, and spot detection. If these vectors are convolved with themselves or with one another, we obtain, among others, the following three vectors, each of length five: $L 5=\{1,4,6,4,1\}$, $S 5=\{-1,0,-2,0,-1\}$, and $E 5=\{-1,-2,0,2,1\}$, which perform local averaging, spot, and edge detection, respectively. The masks used in our analysis are $L 5^{T} E 5$ and $L 5^{T} S 5$. The masks were convolved with each image, and the entropy of the resulting image was calculated.

\section{Fractal Dimension and Lacunarity}

The fractal dimension (FD) is computed based on the concepts of multiresolution image analysis and fractional Brownian motion model (fBMM). The intensity surface of an ultrasonic image can be viewed as the end result of random walks, and fBMM [18] can be used for its analysis. FD and lacunarity (LAC) are the important features that characterize the roughness and granularity of the fractal surface. Given an $M \times M$ image $I$, the intensity difference vector (IDV) is defined as IDV= $[i d(1), i d(2), \ldots i d(s)]$, where $s$ is the maximum possible scale and $i d(k)$ is the average of the absolute intensity difference of all pixel pairs with horizontal or vertical distance $k$. We compute $i d(k)$ as

$$
\begin{aligned}
i d(k)= & \frac{1}{2 M(M-k-1)} \sum_{i=0}^{M-1} \sum_{j=0}^{M-k-1}|I(i, j)-I(i, j+k)| \\
& +\sum_{i=0}^{M-k-1} \sum_{j=0}^{M-1}|I(i, j)-I(i+k, j)|
\end{aligned}
$$

and $D=3-H$, where $D$ is the fractal dimension. The value of $H$ is obtained by using least squares linear regression to estimate the slope of the curve of $i d(k)$ versus $k$ in $\log -\log$ scale. Given a fractal set $A$, let $P(m)$ be the probability that there are $m$ points within a box of size $L$, centered about an arbitrary point of $A$. We have $\sum_{m=1}^{N} P(m)=1$, where $N$ is the number of possible points within the box. The LAC is defined as

$$
\wedge=\left(M_{2}-M^{2}\right) / M^{2}
$$

where $M=\sum_{m=1}^{N} m P(m)$ and $M_{2}=\sum_{m=1}^{N} m^{2} P(m)$.

\section{E. Histogram-Based Features}

The histogram-based features calculated were mean, variance (VAR), coefficient of variation (CV), skewness, kurtosis, and second moment (SM).

Out of the 64 features extracted, the features of GLDM and SGLDM had similar variations. Since computation of SGLDM features is both time- and memory-consuming, we discarded those features. The features that were retained are FD, intercept, LAC, CON, ENT, IDM, ASM, MEAN from GLDM, entropy of Law's textural measures, mean, VAR, CV, skewness, SM, and kurtosis from histogram of the images. We performed Pearson's correlation test on these features to find out which of them are highly correlated with GA. The following six features had a correlation coefficient greater than 0.5: FD, LAC from fractal measures, mean, VAR, CV, and SM calculated from the histogram. The ratios of these features were used as input to the classifiers. It was observed that data sets from both the hospitals exhibited similar behavior.

\section{Classifiers}

The ultrasonic images were classified into mature and immature classes using the following classifiers.

\section{A. Nearest Neighbor Classifier}

This classifies an unknown sample as belonging to the same class as that of the most similar or nearest sample point in the 
training set of data. "Nearest" can be taken to mean the smallest Euclidean distance in the feature space.

\section{B. $k$-Nearest Neighbor ( $k$-NN) Classifier}

There is a possibility of an NN classifier yielding an erroneous decision if the obtained single neighbor is an outlier of some other class. To avoid this and improve the robustness of the approach, the $k$-NN classifier works with $k$ patterns in the neighborhood of the test pattern. In our study, the value of $k$ was chosen to be seven after testing with a number of values of $k$.

\section{Modified k-Nearest Neighbor ( $m k$-NN) Classifier}

In the $k$-NN classifier, though a search in a $k$-neighborhood drives classification, the distance of a template from the test character does not play any role. In an effort to bring about a weighted representation, the $m k-\mathrm{NN}$ classifier or weighted $k$-NN associates a distance-based weight with each prototype member in the $k$-neighborhood. This weighted representation schedule then drives the classification process. The algorithm is as follows.

1) Compute the Euclidean distances between the test (feature) vector and the reference vectors.

2) Sort the reference vectors based on the distances and choose the least distant $k$ patterns.

3) Calculate weight $w_{i}$ associated with each reference pattern $s_{i}$ in the $k$ neighborhood.

4) Find the class that contributes maximum weight in the neighborhood. Declare the test pattern to be belonging to this class.

The weight is calculated as follows. Let $\mathcal{S}=$ $\left\{s_{1}, s_{2}, \ldots, s_{k}\right\}$ be the set of reference patterns in the $k$ neighborhood of the test pattern in the feature space, sorted in increasing order of their distances from the test pattern. Let $\mathcal{X}=\left\{x_{1}, x_{2}, \ldots, x_{k}\right\}$ be the respective distances from the test pattern, where $x_{1}$ is the minimum and $x_{k}$ is the maximum distance. Let $\mathcal{W}=\left\{w_{1}, w_{2}, \ldots, w_{k}\right\}$ be a weight set with $w_{i}$ as the weight assigned to pattern $s_{i}$ based on its distance from the test feature vector, given as

$$
w_{i}=\left(x_{k}-x_{i}\right) /\left(x_{k}-x_{1}\right) .
$$

By testing with different values of $k$, we found that $k=7$ gave a classification accuracy better than any other value.

\section{Multilayer Perceptron (MLP)}

The MLP is a feedforward network, capable of generating nonlinear boundaries. It has been successfully applied to solve some difficult and diverse problems. In our study, a two-layer network was chosen with six input nodes, one hidden layer with three nodes, and two output nodes after exploring the performance of various configurations. The hidden layer had sigmoid activation function, whereas the output nodes had linear activation function.

\section{E. Radial Basis Function (RBF) Network}

The RBF network performs interpolation in a multidimensional space. The RBF network has a high-dimensional hidden layer with Gaussian kernels. In the current study, a network with six input nodes, 15 hidden nodes, and two output nodes was used. The centers of the Gaussian kernels were estimated through $k$-means algorithm.

\section{F. Support Vector Machine (SVM)}

In the SVM, the optimal hyperplane decides the separation between individual classes of patterns. The optimality is in the following sense: the average distance between the hyperplane and the closest training points on both sides is maximal. This aids in maximizing the correct classification rate. Whereas data with linear separability may be analyzed with a hyperplane, linearly nonseparable data are analyzed with kernel functions such as higher order polynomials, Gaussian, and tan-sigmoid. The output of an SVM is a linear combination of the training examples projected onto a high-dimensional feature space through the use of kernel functions. We tested all the kernels mentioned above, and the Gaussian kernel gave the highest classification accuracy. The value of the standard deviation was chosen as 0.2 .

The nearest neighbor, $k$-nearest neighbor [21], and $m k$ nearest neighbor classifiers were implemented in C. For multilayer perceptron and radial basis function [22], we have used Netlab, a package developed by Bishop and Nabney, Neural Computing Research Group, Aston University, U.K. For SVM, we used SVM_TORCH, a software developed by a group at IDIAP, Switzerland [23].

\section{TRAINING AND TEST DATA}

The collected data were grouped into two classes, namely, immature and mature, depending on the GA. Samples from 24 to 34 weeks of gestation were considered to belong to the immature class (possibility of pulmonary risk) and those from 36 to 38 weeks as mature (reduced risk), as pulmonary risk is very rare after 35 weeks of gestation. Three different types of training and test sets were formed from the samples. The first set had 800 training and 200 test samples. Of the training data, 600 belonged to the immature class and 200 to the mature class. The test set contained 150 samples of immature class and 50 of mature class. In the second training set, 400 samples were from 24 to 30 weeks of gestation and 200 from 36 to 38 weeks. In the test set, 200 samples belonged to 32 to 34 weeks of gestation and 50 to 36 to 38 weeks. The third set contained 400 training samples, with an equal number from immature and mature classes. The test set had 100 samples, and an equal number of immature and mature samples were randomly picked from the total data set. The rationale behind the selection of these three training and test sets is explained in the discussion section. In our training, we have considered immature lung classified as immature as true positive (TP) and immature lung classified as mature as false negative (FN).

\section{RESULTS}

Fig. 1 shows the lung and liver areas in the fetal images and the ROIs for extraction of textural features. Fig. 2 gives the descriptive statistical details of the lung-to-liver feature values in the form of boxplots. It also characterizes the variation of the above features with respect to GA. Fig. 3 demonstrates the dynamics of the features, chosen based on the correlation test, as a function of the GA for the lung and the liver. Fig. 3(a) and (b) 

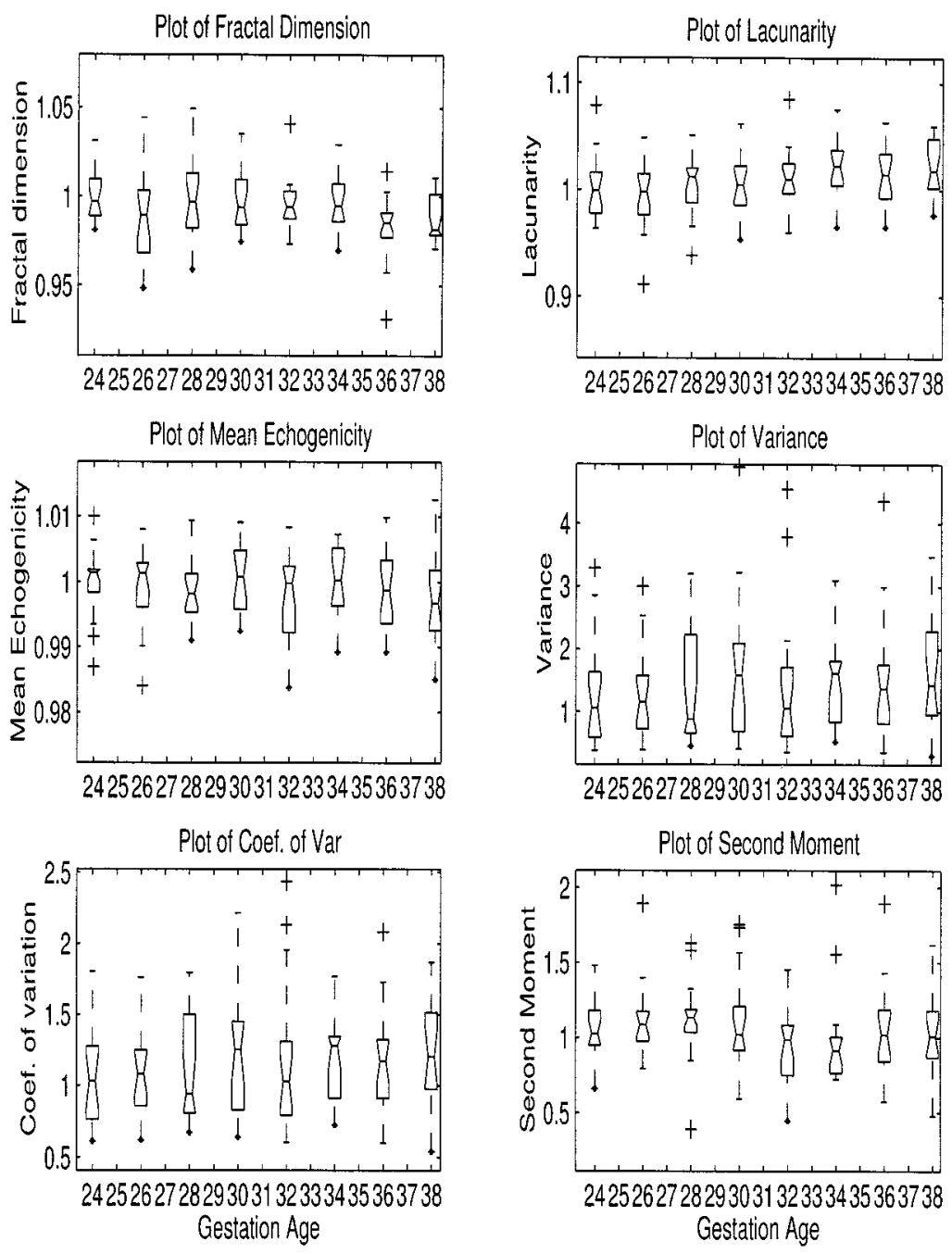

Fig. 2. Plot showing the variation of the ratio of lung-liver feature values with gestation age.

show that the FD and LAC of lung increase as a function of the GA. This is to be expected because the granularity of the cells changes with the GA. Explicitly, the cells of the lung are found elongated during early gestation period, which could give rise to images that are quite smooth, that is, less granular in nature. However, the cells become spherical and fluid-filled [3] toward the term. Further, since the fluid-to-tissue content ratio changes with GA, the diffuse scattering properties also change, leading to more granular images.

Fig. 3(c) shows a decrease in the echogenicity of lung as compared to liver as the GA increases. The echogenicity of the lung is almost the same as the echogenicity of the liver at early GA. Thus, the lung seems to attenuate ultrasound waves more than the liver at later GAs (see [6]). The variance of the gray values of the lung [Fig. 3(d)] has an upward trend with GA, whereas that of the liver remains relatively unchanged throughout the period. The plot of CV [Fig. 3(e)] shows a similar trend as that of the VAR, and the plot of SM Fig. 3(f) is similar to that of FD. It may be noted that the nature of variation of the features of the liver is, in most cases, similar to that of the lung.

Table I shows the results of classification. In all the cases, we have fewer false negatives, which is a preferred outcome. The results indicate the consistency of classification.

\section{DISCUSSION}

In the area of fetal monitoring, a reliable method to determine lung maturity is very essential. The use of ultrasound to study fetal lung maturity is advantageous over others because of its noninvasive nature. Traditionally, ultrasound has been used in fetal monitoring to obtain physical measurements of fetal size and placenta condition. In our work, pulmonary risk assessment based on ultrasound textural features has given encouraging results. Since in all the cases the lung and the liver have been imaged together, the effects due to the imaging techniques (including the internal processing by the machine) must affect both of the regions similarly, and thus must not cause any variations on the textural features of the lung and liver differentially.

We have considered three different training and test sets, as explained in the earlier section. This formulation is to test the generalization and adaptability of the classifiers. The first training set is more biased toward immature class. We need our classifiers to be more sensitive and specific for immature class, because classification of immature class as mature is less desirable. If a mature lung is classified as immature, the problem is not very serious because adequate care would be taken. The classifiers' accuracy for the training and test sets is 

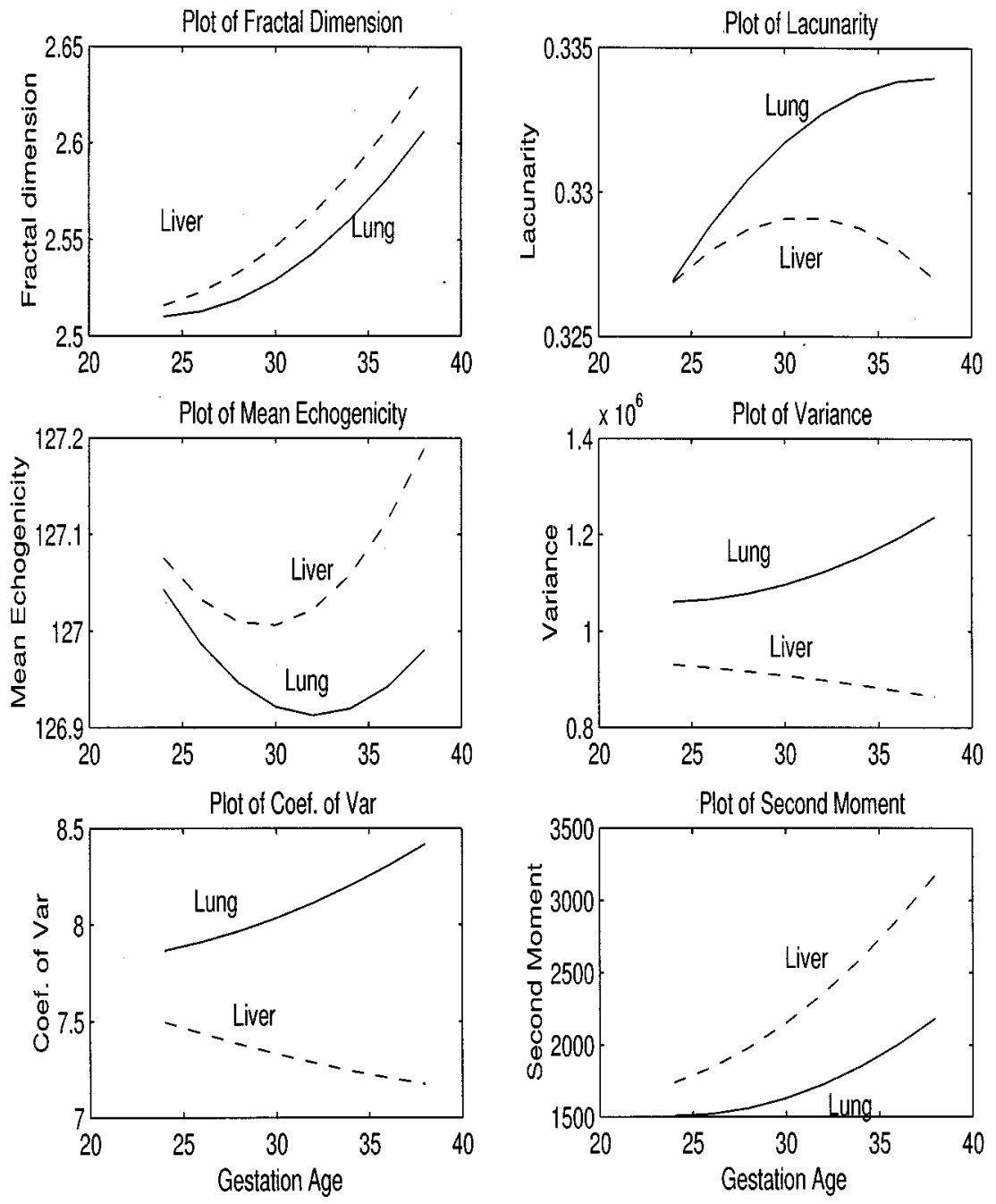

Fig. 3. Plot showing the variation of various features of lung and liver with respect to gestation age.

given in Table I. It may be noted that all the classifiers have almost comparable accuracy of classification, with $\mathrm{NN}$ and $m k$-NN having a little edge over the others. The number of false negatives is low for every classifier. The results show a high degree of specificity of the classifiers to the immature class.

In the second case, the training set did not contain any sample from 32 to 34 weeks, and the test set did not contain any sample from GAs below 32 weeks. This step was undertaken to ensure that the classifier is not biased by data, which could belong to either of the classes. Maturity does not occur before 32 weeks for a normal fetus, whereas it is guaranteed beyond 36 weeks [24]. Thus, the testing of images from the intervening period is likely to throw light on the transition period. An increase in the number of false negatives is seen in this case (see Table I). This is because we have assumed that the data correspond to the immature class, whereas in reality, some of them could actually have transited to the mature class. As seen from the results, the NN classifier and its variants have performed poorly when compared to other classifiers, showing their poor generalization capability.

In the third set, we had an equal number of samples from both classes in order to have an unbiased training of the classifiers.
From Table I, we see that even in the case of unbiased training, the classification results are very consistent, with few false negatives. In fact, the results are very close to the biased training, and in the case of RBF and SVM classifiers, better than the latter.

The NN and $m k$-NN have performed better where representative samples from all GAs were used. The performance of all the classifiers was similar when trained and tested with samples only from definitely immature ( $\leq 30$ weeks) and mature $(\geq 36$ weeks) classes. When the training and test data contained samples from all the GAs, the neighborhood classifiers performed better. Their accuracy can be attributed to more closeness in the neighborhood relation among the samples at the boundary of the two classes. The boundary samples along with the other samples of the two classes contribute to the greater accuracy of $\mathrm{NN}$ and $m k$-NN classifiers.

The classification accuracy for $k$-NN and $m k$-NN was computed for various values of $k$ starting from $k=2$ for the training and test sets. The classification accuracy decreased with increasing $k$ up to $k=6$, started increasing from $k=7$, and decreased once again for $k>8$. Based on this test, the value of $k=7$ was selected.

Some of the earlier studies [2], [4]-[7], [9] have established the usefulness of ultrasound examinations in predicting pul- 
TABLE I

CONFUSION MATRIX OF VARIOUS Classifiers FOR TEST SETS 1, 2, AND 3 (I: ImMature Class, M: Mature Class, CA: Classification ACCURACY FOR TEST SET, AND FN: NUMBER OF FALSE NEGATIVES)

\begin{tabular}{|c|c|c|c|c|c|c|c|}
\hline \multirow{4}{*}{ Classifiers } & \multirow{4}{*}{$\begin{array}{c}\text { Correct } \\
\text { Class }\end{array}$} & \multicolumn{6}{|c|}{ Classifier output } \\
\hline & & \multicolumn{2}{|c|}{ Test set-1 } & \multicolumn{2}{|c|}{ Test set-2 } & \multicolumn{2}{|c|}{ Test set-3 } \\
\hline & & $\mathrm{I}$ & M & I & $\mathrm{M}$ & I & $\mathrm{M}$ \\
\hline & & 150 & 50 & 200 & 50 & 50 & 50 \\
\hline \multirow{4}{*}{ NN } & I & 145 & 5 & 170 & 30 & 46 & 4 \\
\hline & M & 3 & 47 & 33 & 17 & 0 & 50 \\
\hline & $\mathrm{CA}$ & \multicolumn{2}{|c|}{$96 \%$} & \multicolumn{2}{|c|}{$74.8 \%$} & \multicolumn{2}{|c|}{$96 \%$} \\
\hline & FN & \multicolumn{2}{|c|}{5} & \multicolumn{2}{|c|}{30} & \multicolumn{2}{|c|}{4} \\
\hline \multirow{4}{*}{ k- NN } & I & 142 & 8 & 164 & 36 & 44 & \\
\hline & M & 30 & 20 & 30 & 20 & 16 & 34 \\
\hline & $\mathrm{CA}$ & \multicolumn{2}{|c|}{$81 \%$} & \multicolumn{2}{|c|}{$73.6 \%$} & \multicolumn{2}{|c|}{$78 \%$} \\
\hline & FN & \multicolumn{2}{|c|}{8} & & & \multicolumn{2}{|c|}{6} \\
\hline \multirow{4}{*}{ mk- NN } & I & 147 & 3 & 170 & 30 & 46 & 4 \\
\hline & $\mathrm{M}$ & 15 & 35 & 14 & 36 & 5 & 45 \\
\hline & $\mathrm{CA}$ & \multicolumn{2}{|c|}{$91 \%$} & \multicolumn{2}{|c|}{$82.4 \%$} & \multicolumn{2}{|c|}{$91 \%$} \\
\hline & FN & \multicolumn{2}{|c|}{3} & 3 & & \multicolumn{2}{|c|}{4} \\
\hline \multirow{4}{*}{ MLP } & I & 150 & 0 & 187 & 13 & 46 & \\
\hline & $\mathrm{M}$ & & 13 & 37 & 13 & 16 & 34 \\
\hline & $\mathrm{CA}$ & \multicolumn{2}{|c|}{$81.5 \%$} & \multicolumn{2}{|c|}{$80 \%$} & \multicolumn{2}{|c|}{$80 \%$} \\
\hline & FN & \multicolumn{2}{|c|}{0} & & & \multicolumn{2}{|c|}{4} \\
\hline & I & 142 & 8 & 190 & 10 & 47 & \\
\hline & M & & 20 & & 7 & 14 & 36 \\
\hline RBF & $\mathrm{CA}$ & & & 78 & & & \\
\hline & FN & & & & & & \\
\hline & I & 142 & 8 & 165 & 35 & 47 & 3 \\
\hline & M & 23 & 27 & 22 & 28 & 10 & 40 \\
\hline SVM & $\mathrm{CA}$ & 84 & & 77 & & & \\
\hline & FN & & & & & & \\
\hline
\end{tabular}

monary maturity with various degrees of accuracy by using various features like placenta grading, physical measurements of fetal size, echogenicity of the lung and liver region, attenuation of frequencies in lung and liver region, and shift in the RF frequency spectrum during the process of maturation. We have used textural features as a measure of pulmonary maturation, which has yielded an accuracy of classification from $73 \%$ to $96 \%$. To the best of the authors' knowledge, this seems to be the first ever attempt to classify fetal lung maturity in terms of textural features of the ultrasound image.

\section{CONCLUSION}

In this paper, we have shown that the textural features are better indicators of the histological changes, compared to the study of only the echogenicity. Based on the obtained results, it appears that a fairly accurate decision about the maturity of the fetal lung can be made based on the characteristics of the ultrasound images. This result is worth investigating further because of its clinical ramifications. A complete sonographic analysis, which combines the above textural features with parameters such as fetal BPD, PG, femur length, head circumference, and the abdominal circumference, could possibly enhance the prediction accuracy. Also, an analysis of data from high-risk pregnancies (mothers with hypertension or diabetes mellitus) could be used to further validate the prediction of maturity using sonographic features. Further investigation of textural features of ultrasound with biochemical tests will help to establish the validity of the method and eliminate the use of invasive tests for fetal pulmonary maturity analysis.

\section{REFERENCES}

[1] L. B. J. Byrne. (2000) Prenatal Diagnosis. tech. rep., University of Utah, Salt Lake City, UT. [Online]. Available: http://telpath2.med.utah.edu/WebPath/tutorial/prental/prenatal.html

[2] M. Feingold, J. Scollins, C. CetrulO, and D. Koza, "Fetal lung to liver reflectivity ratio and lung maturity," J. Clin. Ultrasound, vol. 15, pp. 384-387, 1987.

[3] Thieme, M. Johnson, H. Silvers, Banjavic, and Carson, "Sonographic identification of lung maturation in the fetal lamb," Invest. Radiol., vol. 18 , pp. 18-26, 1983.

[4] W. J. Garrett, P. S. Warren, and R. H. Picker, "Maturation of the fetal lung, liver and bowel," Radiology, vol. 155, pp. 473-475, 1985.

[5] P. D. Cayea, D. C. Grant, P. M. Doubilet, and T. B. Jones, "Prediction of fetal lung maturity: Inaccuracy of study using conventional ultrasound instruments," Radiology, vol. 155, pp. 473-475, 1985.

[6] D. M. Benson and L. D. Waldroup, "Ultrasonic tissue characterization of fetal lung, liver and placenta for the purpose of assessing fetal maturity," J. Ultrasound Med., vol. 2, pp. 489-494, 1983.

[7] M. Podobnik, B. Brayer, and B. Ciglar, "Ultrasonic fetal and placenta tissue characterization and lung maturity," Int. J. Gynecol. Obstet., vol. 54, pp. 221-229, 1996.

[8] R. A. Petrucha, S. H. Golde, and L. D. Platt, "The use of ultrasound in the prediction of fetal pulmonary maturity," Amer. J. Obestet. Gynecol., vol. 144, no. 8, pp. 931-934, 1982.

[9] C. Sohn, W. Stolz, A. S. Gast, and G. Bastert, "Ultrasound diagnosis of fetal lung maturity," Zentralbl Gynakol, vol. 117, no. 3, pp. 138-143, 1995.

[10] C. Sohn, W. Stolz, A. S. Gast, G. Bastert, and D. von Fournier, "Sonographic diagnosis of fetal lung maturity-A new method," Zentralbl Gynakol, vol. 113, no. 20, pp. 1114-1121, 1991.

[11] C. Sohn, W. Stolz, A. S. Cast, and G. Bastert, "Ultrasound diagnosis of fetal lung maturity," Zentralbl Geburtshilfe Perinatol, vol. 196, no. 23, pp. 55-60, 1992.

[12] U. Raeth, D. Schlaps, B. Limberg, I. Zuna, A. Lorenz, G. van Kaick, W. J. Lorenz, and B. Kommerell, "Diagnostic accuracy of computerised b-scan texture analysis and conventional ultasonography in diffuse parenchymal and malignant liver disease," J. Clin. Ultrasound, vol. 13, pp. 87-99, 1985 .

[13] H. Huisman and J. Thijssen, "Adaptive texture feature extraction with application to ultrasonic image analysis," Ultrasonic Imag., vol. 20, pp. 132-148, 1998

[14] K. N. Bhanu Prakash, A. G. Ramakrishnan, S. Suresh, and W. P. C. Teresa, "Fetal lung maturity analysis using sonogram textural feature," in Proc. Symp. Biomed. Eng. 2000 (BARC), Mumbai, India, Jan. 2000, pp. $155-158$.

[15] R. M. Haralick, "Statistical and structural approaches to texture," Proc. IEEE, vol. 67, no. 5, pp. 304-322, 1979

[16] R. W. Conners and C. A. Harlow, "A theoretical comparison of texture algorithms," IEEE Trans. Pattern Anal. Machine Intel., vol. PAMI-2, no. 3, pp. 204-222, 1980.

[17] K. I. Laws, "Texture energy measures," in Proc. Image Understanding Workshop, 1979, pp. 47-51. 
[18] J. M. Keller, S. Chen, and Crownover, "Texture description and segmentation through fractal geometry," Comput. Vision, Graph. Image Process., vol. 45, pp. 150-166, 1989.

[19] S. Dudani, "The distance-weighted $k$-nearest neighbor rule," IEEE Trans. Syst., Man, Cybern., vol. SMC-6, pp. 325-327, Apr. 1976.

[20] V. Vapnik, The Nature of Statistical Learning Theory. New York: Springer, 1995.

[21] R. Duda and P. Hart, Pattern Classification and Scene Analysis. New York: Wiley, 1973.

[22] S. Haykin, Neural Networks: A Comprehensive Foundation. Englewood Cliffs, NJ: Prentice-Hall, 1999, ch. 4-6.

[23] R. Collobert and S. Bengio, "On the convergence of SVMTorch: An algorithm for large scale regression problems," Tech. Rep., Dalle Molle Institute for Perceptual Artificial Intelligence, Martigny, Switzerland, 2000.

[24] L. Gluck and M. V. Kulovich, "Fetal lung development: Current concepts," Ped. Clin. North Amer., vol. 20, pp. 275-292, 1973.

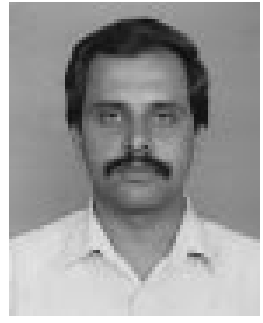

K. N. Bhanu Prakash received the M.E. degree from the Indian Institute of Science, Bangalore, in 1991, where he currently is pursuing the Ph.D. degree.

His doctoral thesis involves processing of ultrasound and MR images. He was a Quality Control Engineer with Bells Control Ltd. briefly before joining the Faculty of Sri Jayachamarajendra College of Engineering, Mysore, India. He was a Visiting Scholar at the Department of Electrical Engineering, University of Kaiserslautern, Germany, during 1997. His interests include image processing, pattern recognition, and neural networks.

Mr. Bhanu Prakash received a National Merit Scholarship during his undergraduate studies.

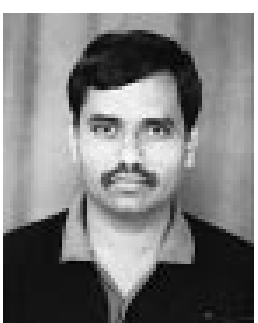

Society of India.

Dr. Ramakrishnan is a Senior Member of EMBS and SP societies and has received the Thangam Vasudevan Award (Indian Association of Biomedical Scientists), Sir Andrew Watt Kay Young Researcher's Award (Royal College of Physicians and Surgeons, Glasgow), and Young Investigator Award (Department of Science and Technology, Government of India).

S. Suresh is a specialist in medical untrasound with extensive knowledge and skills in various applications. He has done pioneering work in India in the field of prenatal diagnosis, fetal therapy, and interventional ultrasound. He developed the Mediscan Systems Diagnostic Ultrasound Research and Training Centre, Madras, India, in 1982, which has grown into the largest dedicated ultrasound center in India. He has conducted training courses for physicians since 1985 and has conducted several untrasound courses in Malaysia for the last four years. He has organized international conferences on ultrasound and has authored about 30 publications in international and national journals and has lectured at centers around the world on a wide range of topics pertaining to ultrasound. He developed the Fetal Care Research Foundation and its affiliated organizations, the Surendra Genetic Laboratory and the Center for Perinatal Pathology for further research in the field of congenital anomolies of the fetus. Under its auspices, for the first time in India, a birth defects registry has been established which is collecting data on prevalence of birth defects in Chennai. His other interests include amateur radio communication and applications of computers to medicine and photography.

Teresa W. P. Chow, photograph and biography not available at the time of publication. 